\title{
Towards an optimal estimation of the SMOS antenna-frame systematic errors
}

\author{
Jérôme Gourrion, Sébastien Guimbard, Marcos Portabella and Roberto Sabia
}

\begin{abstract}
After 2.5 years of the Soil Moisture and Ocean Salinity (SMOS) mission, the characterization of residual instrumental systematic errors in the measured brightness temperatures $\left(T_{B}\right)$ is still rather poor. This in turn negatively impacts the sea surface salinity retrievals and, as such, notably limits the mission's success. The error mitigation methodology currently used operationally, the so-called Ocean Target Transformation (OTT), mixes both instrumental and model-induced errors. In this work, it is proposed to distinguish errors by their type of impact on the $T_{B}$ images: mean brightness level, incidence and azimuth angle dependence. A new approach to characterize the azimuthdependent errors is proposed. First, a careful data selection strategy is applied. Then an empirically fitted model, which only accounts for the $T_{B}$ incidence angle dependence, is subtracted from the mean $T_{B}$ images of the selected datasets to estimate the systematic antenna frame errors. The robustness of this methodology is assessed through the estimated anomaly pattern stability when computed for different geophysical conditions, periods of time, and latitudinal bands. The residual variability ranges from $0.03 \mathrm{~K}$ to $0.14 \mathrm{~K}$, whereas the OTT variability is about $0.5 \mathrm{~K}$. The new method is forward model independent and generic. It can therefore be applied to estimate the antenna frame systematic errors over land and ice. Moreover, it proves to be very effective in separating different sources of error and can therefore be used to further characterize other error components and improve the various SMOS forward model terms.
\end{abstract}

\section{Index Terms}

Remote sensing, Soil Moisture and Ocean Salinity (SMOS), radiometer, error correction, interferometer, sea surface salinity.

\section{INTRODUCTION}

The Soil Moisture and Ocean Salinity (SMOS) satellite was launched on November 2, 2009 in the framework of the European Space Agency's (ESA's) Earth Explorer opportunity missions. Over the oceans, sea surface salinity (SSS) is retrieved on a global basis with a spatio-temporal sampling appropriate for ocean dynamics and Earth water cycle studies [1], [2]. The single payload onboard SMOS is the Microwave Imaging Radiometer using Aperture Synthesis (MIRAS), a novel fully-polarimetric L-band radiometer which estimates the brightness temperature $\left(T_{B}\right)$ by means of two-dimensional aperture synthesis interferometry. It consists of a Y-shaped set of 72 receivers

J.Gourrion, S.Guimbard and M.Portabella are with the Physical Oceanography Department and SMOS-BEC, Institut de Ciències del Mar (ICM/CSIC), Barcelona, Spain. R.Sabia is with the European Space Agency (ESA-ESRIN), 00044 Frascati, Italy. Contact: gourrion@cmima.csic.es, gourrion@gmail.com 
[3]. The MIRAS measurement processing starts at visibility level, i.e. correlations of the electromagnetic signal measured by pairs of receivers. Once the so-called visibilities are calibrated, corrections for aliasing, foreign source contributions (sun, moon and radio-frequency interference), and a-priori knowledge of instrument model errors (Flat Target Transformation - FTT, see [4]) may be applied. Then, using a mathematical model of the instrument, the reconstruction procedure allows recovery of a $T_{B}$ image [5].

During the first 6 months after launch, the SMOS satellite underwent the In-Orbit Commissioning Phase (IOCP), a customary calibration and checkout period. Despite the substantial progress in the instrument calibration, improvement in the detection and mitigation of instrument and image reconstruction $T_{B}$ biases over the oceans remained critical [6]. Indeed, systematic antenna-based error patterns are detected in SMOS $T_{B}$ measurements, as expected from pre-launch simulations [7], [8]. Such error patterns have typical amplitudes of $\pm 5 \mathrm{~K}$ [9]. In the sea surface incidence/azimuth frame centered on the target, the sea surface first-Stokes brightness temperature is expected to vary essentially along the incidence angle dimension. The aforementioned errors introduce systematic azimuthal inconsistency between measurements acquired at the same incidence angle but at different locations in the field of view (FOV). Note that such errors are hereafter referred to as azimuthal anomalies. When retrieving geophysical parameters, these systematic errors are combined with imperfections in both the emissivity forward model and the inversion methodology, resulting in strong spatio-temporal SSS biases which exceed mission requirements [10]. The context of the present study is the definition of an adequate methodology to empirically characterize the systematic component of such error patterns.

Separate characterization of the potential error sources over the ocean is a non-trivial challenge as they all have different characteristics in terms of spatial and temporal structure. Residual visibility calibration errors may mainly arise from the characterization of both the Power Measurement System (PMS) and the antenna losses [11]. For all receivers, PMS offset (gain) fulfils stability specifications over one-week (two-month) periods. In the SMOS routine calibration plan, such periods constrain the acquisition frequency of the specific calibration sequences as well as the frequency of the gain and offset estimation and their update in the processing chain. Under the reasonable assumption that residual PMS errors meet stability requirements (0.1\%, see [11]) for one-week (two-month) periods, the impact of the latter errors on $T_{B}$ is expected to be slowly varying in time and to remain lower than a few tenths of a Kelvin, more than an order of magnitude smaller than the errors of interest in the present study ( $\pm 5 \mathrm{~K}$, see above). Characterization of antenna losses is still an on-going task of the SMOS calibration team. Its imperfection may lead to errors in the antenna power gain. In addition to a seasonal scale, [11] show that errors in the antenna loss model induce discrepancies between ascending and descending passes, i.e. orbital-scale errors. As such, in the case of temporally averaged brightness temperature measurements, it is expected that the antenna loss errors essentially produce short-term variations in the mean brightness level. The instrument model used for image reconstruction is a potential source of error in the SMOS $T_{B}$ images. The Flat Target Transformation (FTT, see [4]) is a technique to correct for such an error. It is based on the comparison of measured and expected sky visibilities, where the corresponding scenes are acquired during specific platform maneuvers to observe nearly-homogeneous domains of the celestial sphere. Unfortunately, this method is not expected to be perfect when applied to the ocean surface 
due to its natural anisotropic emissivity described by the Fresnel reflection coefficients. Inconsistencies likely exist between actual in orbit gain and phase antenna patterns and those measured on ground [11]. Such an imperfect knowledge of the individual antenna gain patterns induces rather time-stable spatial ripples in the $T_{B}$ images. Errors coming from the sky alias removal should be stable-in-time and located in the outer part of the FOV. Furthermore, issues related to the sharp contrast near land/sea boundaries can be avoided through selection of purely oceanic scenes. All of these error sources degrade the comparability level within a set of observations of the same target acquired at different time or FOV locations. To successfully retrieve SSS, these inconsistencies have to be corrected before inversion. Naturally, a primary task to increase the data consistency is to remove the discrepancy between data acquired at different FOV locations in the antenna reference frame. Indeed, if such an objective is achieved, the characterization of both spatio-temporal instrumental and model biases should be more robust since a much larger consistent dataset can be used and a higher statistical significance level can be reached.

A specific strategy is required to characterize these antenna frame errors. Indeed, in addition to instrumental and reconstruction errors, a SMOS dataset contains both random and environmental ${ }^{1}$ variability. A central point in the estimation of systematic errors from such a dataset is the reduction of the variability since the antenna pattern related errors are expected to be constant in time. The random part of the SMOS image variability is caused by the intrinsic radiometric Gaussian noise which can be reduced by simple averaging of a large number of independent scenes. In contrast, the geophysical variability is, in general, not Gaussian, and specific strategies need to be used to avoid propagating geophysically-related biases into the instrumental bias pattern estimate.

A first commonly-used option involves the use of a forward model of the observations, in this case, a radiative transfer model for the sea surface emission and its propagation up to the instrument level. The principle consists of simulating the geophysical part of the L-band radiation variability, as sensed by the instrument, using auxiliary information (dielectric constant, roughness, atmospheric and reflected celestial contributions, as well as climatological salinity) to correct the data by using the mean departure between data and model, rather than using data exclusively. The SMOS operational processing chain includes a systematic error correction procedure based on this idea [9]

$$
O T T(\xi, \eta)=<T_{B}(\xi, \eta)-T_{B}^{m o d e l}(\xi, \eta)>
$$

If such a strategy is applied periodically, any possible slowly-varying temporal offset in the reconstructed images is filtered out, preventing the separation of geophysical and non-geophysical components of such a signal. Furthermore, a critical assumption of such an approach is that the emissivity model is perfect. Gourrion et al. [12] show that model imperfections and auxiliary parameter errors may negatively impact the corrected brightness temperatures, leading to a $0.5 \mathrm{~K}$ variability of the corresponding error pattern.

A second option deals with the selection of a subset of data with consistent geophysical conditions. In this case, the distribution of auxiliary geophysical parameters may differ within the FOV but should lead to a nearly homogeneous mean impact on the brightness temperatures at a given incidence angle. Departure from such behavior

\footnotetext{
${ }^{1}$ to be understood as the sum of geophysical and external source contributions
} 
may then be interpreted in terms of non-geophysical variability, in the limit of the selection criteria accuracy. At each location in the field of view, the error pattern may therefore be obtained as the mean departure of the brightness temperature from a characteristic reference value that only depends on the surface incidence angle.

In this paper, the second approach is used to characterize the systematic errors in the antenna frame. A recent work [13] proposes to estimate the antenna frame systematic errors, with a similar approach to that proposed in this paper. An essential difference concerns the variability reduction strategy. In [13], both the data selection performed on a geographical and temporal basis and the correction from an ideal $T_{B}$ dependence on incidence angle, which is estimated on a snapshot basis by means of a polynomial regression, likely contribute to obtain a non-Gaussian $T_{B}$ distribution at each antenna frame grid point. As a result, the error patterns estimated from different datasets may vary by more than $0.5 \%$ i.e. more than $0.5 \mathrm{~K}$ in typical oceanic scenes (see Figure 4 in [13]). In this paper, the data selection focuses on reducing the non-Gaussian component of the $T_{B}$ distribution, which is associated with the geophysical variability. Moreover, to further benefit from the data selection approach, the $T_{B}$ adjustment with incidence angle is perfomed over a mean $T_{B}$ scene rather than at each snapshot. In this paper, it is shown that the new approach leads to an estimated error pattern variability of the order of $0.1 \mathrm{~K}$, i.e., 5 times smaller than that achieved by the approach in [13].

In section II, a data selection procedure based on both instrumental and environmental criteria is implemented. In section III, a simplistic and robust empirical model depending only on incidence angle is adjusted to the dataset to reproduce the mean incidence angle dependence of the reconstructed images. It is expected that the departure from this reference model essentially contains the azimuthal anomalies while the reference model reproduces both the homogeneous part and the incidence angle dependence of the observations. In section IV, the effectiveness of the proposed method is assessed in terms of stability of the estimated error pattern. Section V draws the main conclusions.

\section{DATA SELEction}

The objective of the data selection step is to build a dataset for which the measured brightness temperatures follow the expected behaviour of the surface emission, i.e. variations essentially described by the Fresnel reflection coefficients and surface incidence angle changes. Then, the expected variations of the mean brightness temperature (essentially incidence angle dependent and azimuth angle independent) can be isolated from the unexpected ones (called "azimuthal" by contrast, the azimuth isolines being defined as the lines perpendicular to surface incidence angle isolines, as described in Section III). Due to noticeable dataset thinning during data selection, this approach requires the original dataset to be substantially larger than for the previous approach to reach a comparable noise reduction level.

First, the impact of instrumental errors must be addressed. The contribution of the slowly-evolving residual PMS and antenna loss model errors is controlled through limiting the temporal extent of the dataset. An 18-day period is chosen, corresponding to a quasi-perfect subcycle of SMOS orbit which additionally permits the reduction of the risk of potential sampling artifacts. Also, the impact of orbital-scale antenna loss model errors is controlled by 
selecting data from ascending passes only and limiting the latitudinal extent.

In the following, the impact of environmental contributions is addressed. The expected variability of the SMOS L-band brightness temperature may be described as follows:

$$
\mathbf{T}_{f w m}^{H, V}=\left(\mathbf{T}_{\text {surf }}^{H, V}+\mathbf{R}^{H, V} \mathbf{T}_{d}+\mathbf{T}_{s k y}^{H, V} e^{-\tau}\right) e^{-\tau}+\mathbf{T}_{u}
$$

where $H$ and $V$ denote horizontal and vertical linear polarization states, respectively, $\mathbf{T}_{u}$ the upwelling brightness of the atmosphere, $\mathbf{R}$ the reflectivity of the rough sea surface, $\mathbf{T}_{d}$ the downwelling atmospheric brightness temperature, $\mathbf{T}_{s k y}$ the scattered celestial sky noise and $\tau$ the opacity of the atmosphere. In [14] Table III, it is shown that the atmosphere contributes significantly to the effective emissivity but little to its variability. As a consequence, this analysis assumes that the intrinsic emissivity of the sea surface (combined dielectric and roughness contribution,

$\mathbf{T}_{\text {surf }}^{H, V}$ ) and the reflected celestial signal $\mathbf{T}_{s k y}^{H, V}$ are the dominant contributions to the observed geophysical variability in the $H, V$ surface polarization frame. This section presents a data selection strategy to reduce the dispersion induced by such contributions. Additional modification of the signal as observed in the $X, Y$ antenna polarization frame arises in the ionosphere, where Faraday rotation of the electromagnetic fields around the propagation direction may occur, i.e. redistribution of energy among the field components. Recalling that the present study is essentially methodological, it is chosen to avoid such a component of variability by restricting the analysis to half of the first Stokes vector component (S1) which is related to the total energy content of the received waves.

\section{A. Celestial reflections}

Celestial emissions at L-band may be considered as the sum of contributions of nearly homogeneous cosmic background radiation (of the order of $3 \mathrm{~K}$ ), point sources and the Milky Way. They are reflected by the sea surface following Fresnel laws, modified to account for surface roughness effects. The signal emitted by the galaxy may exceed $10 \mathrm{~K}$ locally, inducing spatial gradients that may be as large as a few K across SMOS brightness temperature images, even after reflection by the surface. Figure 1 displays the estimated reflected signal (assuming a smooth surface) averaged over the alias-free portion of the SMOS field of view. Even if a large range of local geometrical conditions is integrated through the spatial averaging process, such a diagram provides relevant information to qualitatively identify the time periods and geographical area where the instrument is pointing far from the galactic plane and where the $T_{B}$ images may be expected to be little and rather homogeneously contaminated by celestial reflections. Based on this information, various time and latitude windows are identified corresponding to expected low and homogeneous celestial contamination conditions (while already reducing impact of latitudinal and temporal instrumental biases, as described at the beginning of Section II) :

- Nov. $29^{\text {th }}-$ Dec. $16^{\text {th }}$, [35 S - $\left.10 \mathrm{~S}\right]$, ascending passes

- Jun. $10^{\text {th }}-$ Jun. $27^{\text {th }},[55 \mathrm{~S}-35 \mathrm{~S}$, ascending passes 


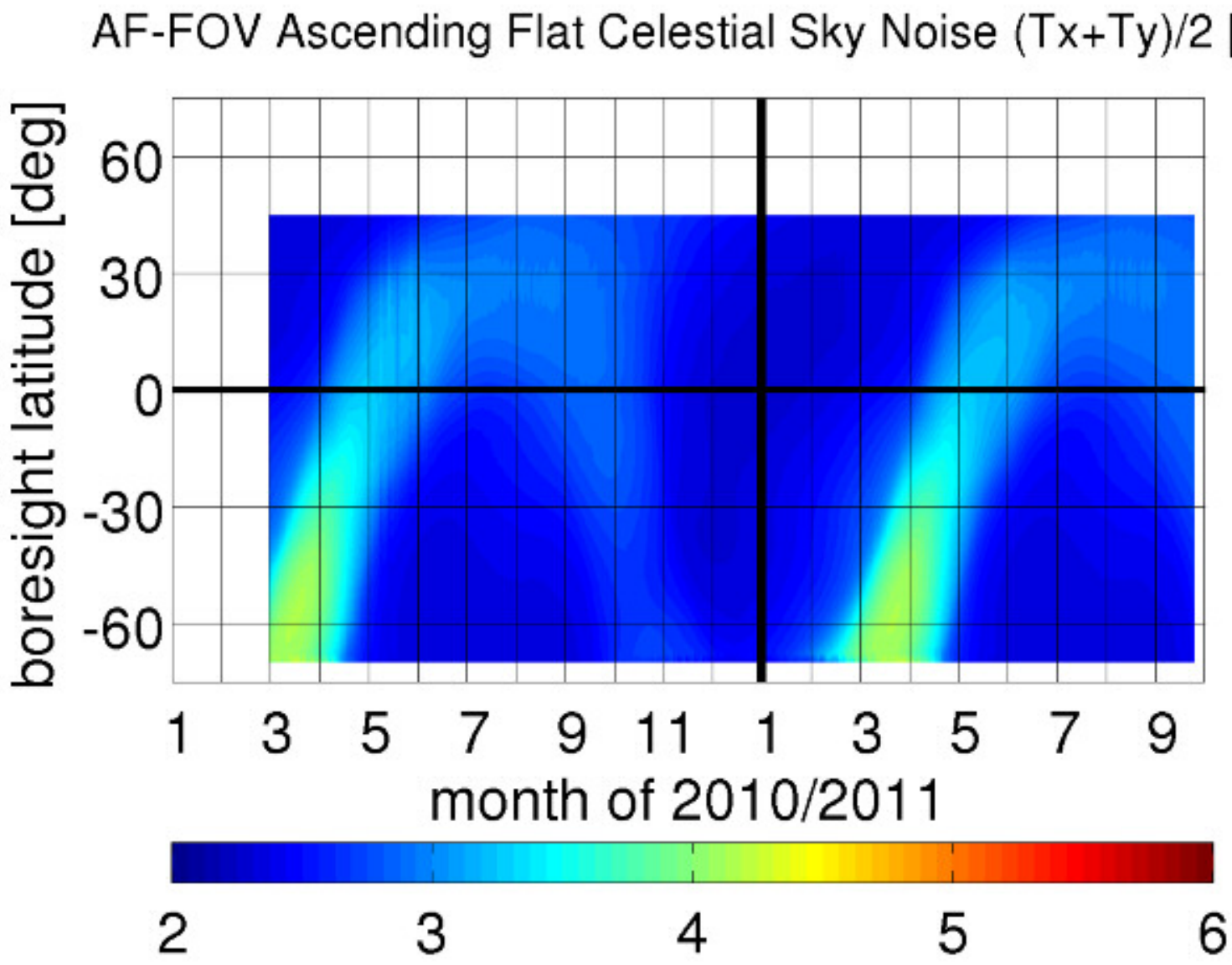

Fig. 1. Hovmuller diagram of the modelled half 1 st Stokes celestial signal as reflected by a smooth sea surface. The results correspond to the geometry of the SMOS platform during ascending passes and are averaged over the entire alias-free field of view. Courtesy of J. Tenerelli.

\section{B. Dielectric and roughness properties}

Sea Surface Temperature (SST) and Salinity (SSS) modify the dielectric properties of the upper layer of the ocean leading to several Kelvin variations of the brightness temperature at L-band [15] (hereafter referred to as KS77). The sea surface roughness induces variability of similar amplitude which may be parameterized, at first order, as a function of local wind speed $U$ [14], typically

$$
\frac{\Delta T_{B}}{\Delta U}=0.5 \mathrm{~K} \cdot \mathrm{m}^{-1} \cdot \mathrm{s}
$$

Following this, and for each dataset specified in the previous section, homogeneous subsets are built to classify the observations as a function of wind speed conditions as provided by the SMOS auxiliary products: $\left|U-U_{0}\right|<\Delta U$ using $U_{0}=\{6,8,10,12\} \mathrm{m} \cdot \mathrm{s}^{-1}$. Allowing a residual roughness-related $T_{B}$ contribution of $0.5 \mathrm{~K}$ and using (3), the selection criterion to be applied on the auxiliary wind speed is $\Delta U=1 \mathrm{~m} \cdot \mathrm{s}^{-1}$. Additionally, for each subset, the expected flat sea brightness temperature $T_{B}^{f}$ as predicted by KS77 is computed using the auxiliary SST and climatological SSS values. The data are further filtered using the criterion $\left|T_{B}^{f}-T_{B}^{f 0}\right|<\Delta T_{B}^{f}$, where $T_{B}^{f 0}$ is the 


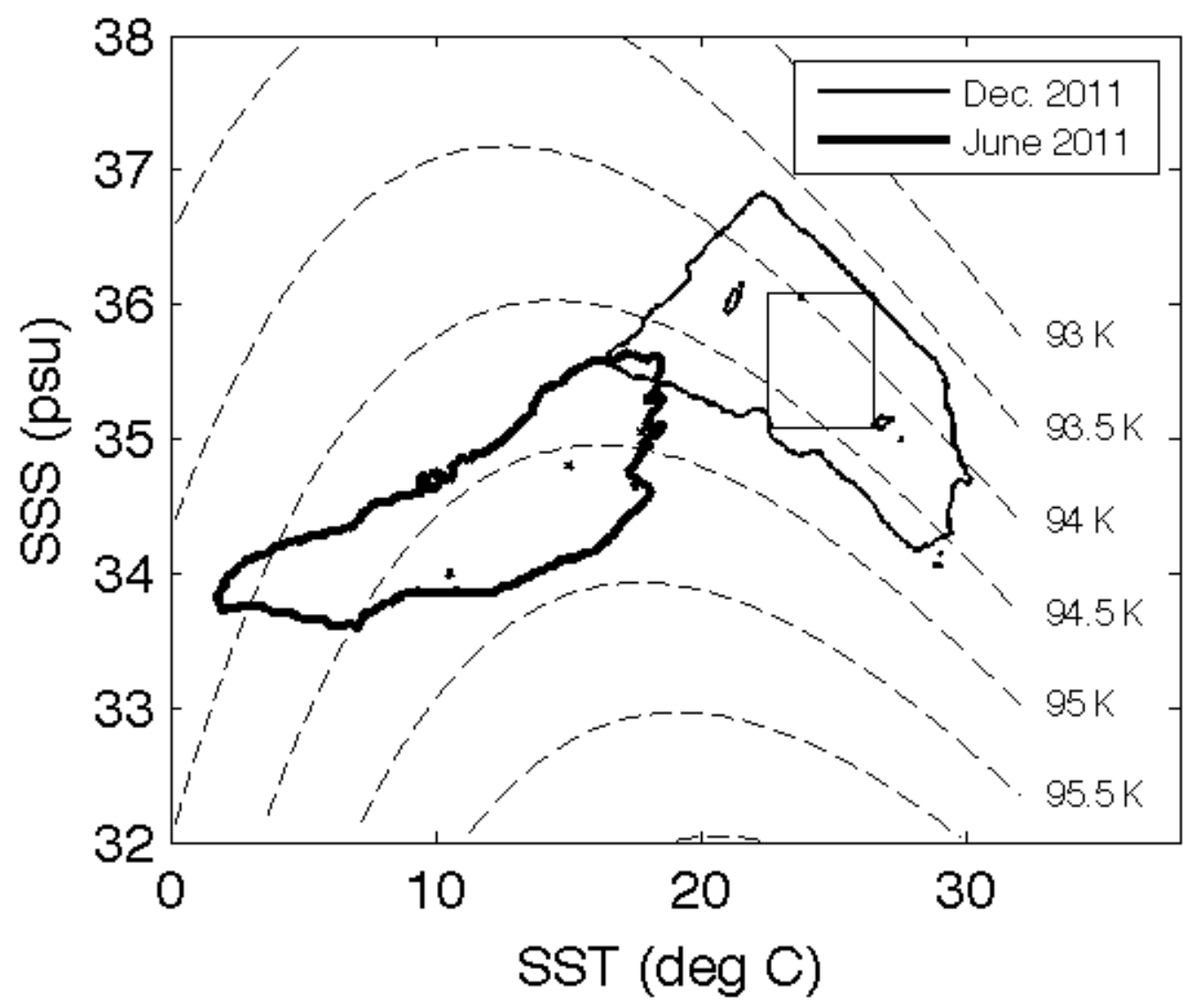

Fig. 2. Contour in the probability density function of auxiliary SST/SSS values corresponding to the $T_{B}$ observations made at $45 \pm 1^{\circ}$ incidence and $8 \pm 1 \mathrm{~m} \cdot \mathrm{s}^{-1}$ wind speed. Contour value is one one-hundredth of the pdf maximum. The thick (thin) line corresponds to the June (December) 2011 dataset. The thin dashed lines correspond to the 93 to $96 \mathrm{~K}$ isolines of half of the first Stokes brightness temperature from a smooth sea surface as expected from KS77. The thin square line indicates the selection domain corresponding to a selection procedure based on SSS and SST thresholds rather than $T_{B}^{f}$.

mean of the $T_{B}^{f}$ distribution (computed separately for different incidence angle bins). Consistent with the allowed roughness residuals, a value $\Delta T_{B}^{f}=0.5 \mathrm{~K}$ is chosen. Note that the exact wind speed and $T_{B}^{f}$ distributions may slightly differ from one incidence angle bin to another.

Figure 2 shows the SST/SSS distribution associated with the data subsets after selection, in the particular case of $45^{\circ}$ incidence angle and $8 \mathrm{~m} \cdot \mathrm{s}^{-1}$ wind speed. The $T_{B}^{f 0}$ value for the June (December) 2011 dataset is 94.87 (94.21) K. The results for other incidence angles or wind speeds are similar. The comparison between the contour and the square domain for the December dataset illustrates the significance of the $T_{B}^{f}$-based selection procedure in terms of sample number and statistical significance increase with regard to a classical SST/SSS strategy with the same constraint on the maximum allowed departure from $T_{B}^{f 0}$. 


\section{Number of samples}

Considering the irregular distribution of geophysical structures within the SMOS field of view, the number of samples selected at each location in the antenna reference frame is not necessarily homogeneous. $\mathrm{N}$ observations are randomly selected at each location where $\mathrm{N}$ is taken as the minimum number of samples among all locations for the corresponding dataset. $\mathrm{N}$ values are provided in Table $\mathrm{I}$. While this step further discards a large number of observations, it homogenizes the significance of the statistical parameters, which is essential when dealing with limited-size datasets.

\section{AZIMUTHAL ANOMALY ESTIMATION}

Once subsets nearly homogeneous in terms of sampled geophysical conditions are built, it is possible to estimate their characteristic variation with incidence angle. Thus, using the selected observations, a mean synthetic image for the S1 parameter is first obtained (see the top left of Figure 3). Second, the mean brightness temperatures are sorted according to incidence angle (see the top right of Figure 3). In contrast with V-and H-pol that are linearly mixed together depending on their location in the field of view and would require rotation correction, no azimuthal variation is expected for $\mathrm{S} 1$ since it is independent of the polarization frame. Third, a $4^{\text {th }}$ order polynomial adjustment is performed to ensure robustness and force the fit to be monotonic as expected.

To avoid discontinuities near the alias boundaries due to imperfect alias removal during the reconstruction procedure ${ }^{2}$, only the points falling inside the circle of radius 0.3 in the director cosine antenna frame (see circle in the top left of Figure 3) are used to adjust the model (while they are all kept when later estimating the error pattern), restricting the range of incidence angles approximately to the interval $[16,60]$ degrees. This implies that between 0 and 16 degrees, the proposed empirical model is the result of an extrapolation procedure. The data have been duplicated symetrically around nadir and defined for negative incidence angle values in order to force the derivative of the function at nadir to be zero, as expected from theory, and constrain the extrapolation. In any case, results are presented only for the larger incidence angles which do not rely on the extrapolated part of the model.

The top left panel of Figure 3 shows an example of an averaged SMOS image at H-pol after rotating the polarization frame to account for geometrical and Faraday rotation effects. It illustrates the presence of azimuthal anomalies, seen here as the coloured variations found along an incidence angle isoline. The top right panel of Figure 3 represents the same average scene but as a function of only incidence angle. An example of the fitted one-parameter empirical model is superposed.

Finally, following (4), for all datasets and all locations in the field of view, the empirical model (Figure 3, top right) is subtracted from the mean value of the SMOS image at that location (Figure 3, top left) to obtain the

\footnotetext{
${ }^{2}$ Note that the outer part of SMOS images is contaminated by aliasing phenomenon since the ratio between electromagnetic wavelength and antenna spacing does not comply the Nyquist criterion. The so-called extended alias-free domain is the part of the field of view free of aliasing once the contribution from the Sky alias has been removed before reconstruction. Unfortunately, alias removal requires a perfect knowledge of the instrument characteristics while, as mentioned earlier, the real antenna diagrams of MIRAS are imperfectly known. The alias removal technique induces errors inside and near the boundaries of the alias contaminated domain.
} 

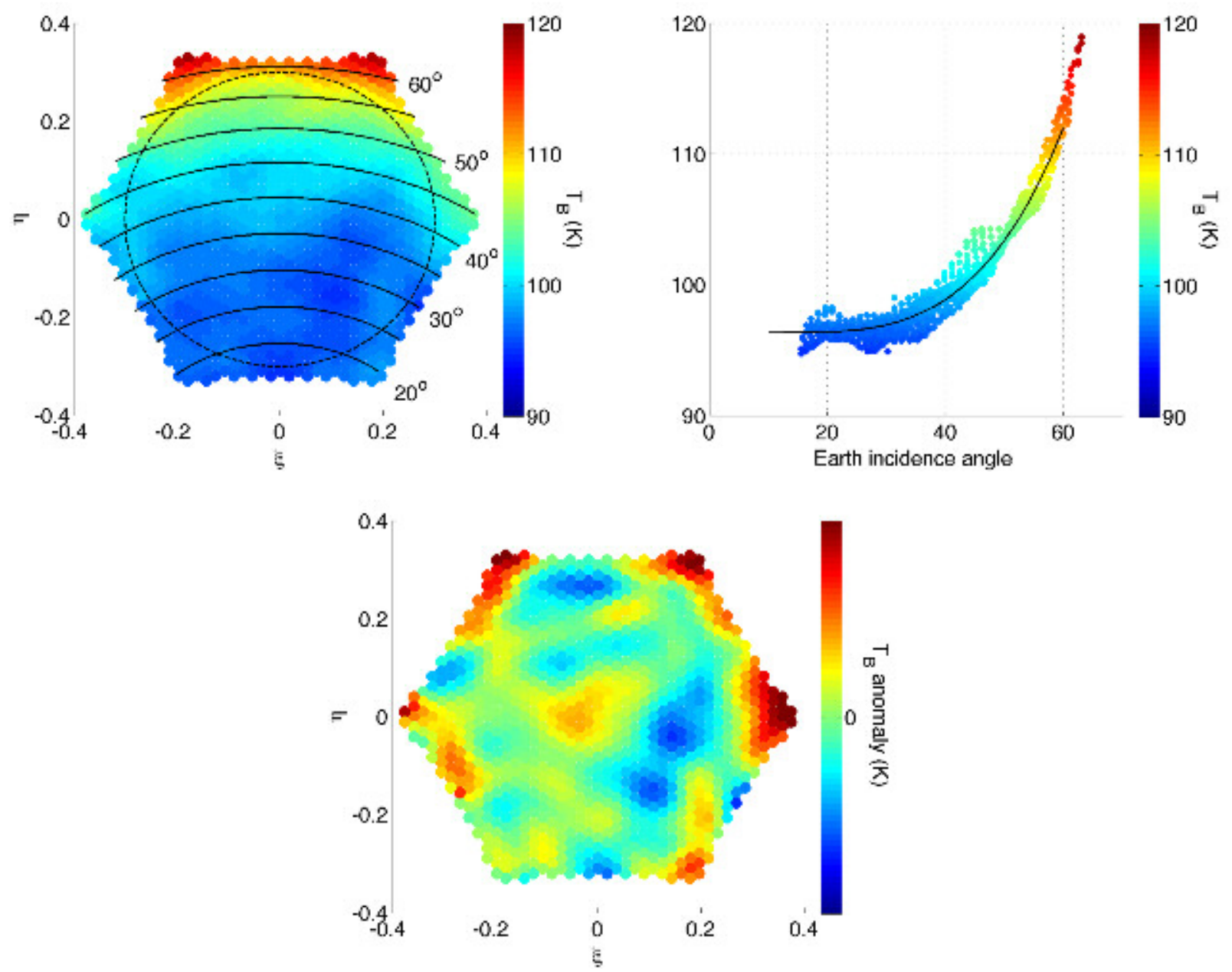

Fig. 3. Top left: average SMOS reconstructed image for half of the first Stokes parameter. Isolines of incidence angle at the Earth surface are superposed. Top right: same data as left plot but represented in a one-dimensional space along incidence angle. An example of empirical polynomial regression as performed in section III is also shown. Bottom: azimuthal anomaly pattern obtained as the difference between the mean image from the left panel and the one-parameter model from the right panel.

azimuthal anomaly pattern (Figure 3, bottom).

$$
O T T(\xi, \eta)=<T_{B}(\xi, \eta)>-T_{B}^{\text {model }}(\theta)
$$

The variability of the pattern among the various subsets is presented in the next section.

\section{RESULTS AND DISCUSSION}

\section{A. Variability of the one-parameter model}

In this section, the variability of the fitted model among the different subsets of geophysical conditions is presented. The variations of the independent empirical models with roughness and dielectric state proxies is presented and compared with predictions from the literature, [14], [15]. 
TABLE I

STATISTICS FROM THE FOUR MAJOR DATASETS USED IN THIS STUDY. U $\mathrm{U}_{0}$ IS THE REFERENCE WIND SPEED IN M.S - 1 USED FOR DATA SELECTION; $\mathrm{N}$ IS THE NUMBER OF OBSERVATIONS USED TO COMPUTE THE ANOMALY PATTERNS AND FORCED TO BE HOMOGENEOUS AT ALL LOCATIONS IN THE FIELD OF VIEW; $\bar{U}, \bar{T}$ AND $\bar{S}$ ARE THE MEAN WIND SPEED, SST AND SSS FURTHER AVERAGED OVER ALL INCIDENCE ANGLE BINS FOR THE SELECTED DATASETS.

\begin{tabular}{|l|rrrr|}
\hline & \multicolumn{4}{|c|}{ June 2010 } \\
\hline $\mathrm{U}_{0}$ & 6 & 8 & 10 & 12 \\
\hline $\mathrm{N}$ & 1677 & 2352 & 2919 & 2896 \\
$\bar{U}$ & 6.1 & 8.0 & 10.0 & 12.0 \\
$\bar{T}$ & 10.7 & 10.2 & 9.8 & 8.9 \\
$\bar{S}$ & 34.42 & 34.43 & 34.41 & 34.343 \\
\hline & \multicolumn{4}{|c|}{ December $\mathbf{2 0 1 0}$} \\
\hline $\mathrm{U}_{0}$ & 6 & 8 & 10 & 12 \\
\hline $\mathrm{N}$ & 4136 & 7057 & 3460 & 409 \\
$\bar{U}$ & 6.1 & 8.0 & 9.7 & 11.8 \\
$\bar{T}$ & 24.2 & 24.1 & 23.1 & 21.8 \\
$\bar{S}$ & 35.47 & 35.51 & 35.61 & 35.33 \\
\hline & \multicolumn{4}{|c|}{ June } \\
\hline $\mathrm{U}_{0}$ & 6 & 8 & 1011 & 12 \\
\hline $\mathrm{N}$ & 1741 & 2311 & 2748 & 3055 \\
$\bar{U}$ & 6.1 & 8.0 & 10.0 & 12.0 \\
$\bar{T}$ & 10.0 & 9.7 & 9.4 & 9.4 \\
$\bar{S}$ & 34.37 & 34.34 & 34.32 & 34.36 \\
\hline & \multicolumn{5}{|c}{ December 2011 } \\
\hline $\mathrm{U}_{0}$ & 6 & 8 & 10 & 12 \\
\hline $\mathrm{N}$ & 4709 & 7198 & 2856 & 351 \\
$\bar{U}$ & 6.1 & 8.0 & 9.7 & 11.7 \\
$\bar{T}$ & 24.2 & 24.1 & 22.9 & 21.5 \\
$\bar{S}$ & 35.42 & 35.55 & 35.68 & 35.49 \\
\hline
\end{tabular}

Figure 4 shows the variation of the empirical one-parameter model (see Figure 3, top right panel) as a function of wind speed (see Figure 2) for the December 2011 dataset. For clarity, only results at a single incidence angle value $\left(30^{\circ}\right)$ are shown. The horizontal axis indicates the mean wind speed for each subset. As the absolute level of the empirical model may be contaminated by a wide range of instrumental and geophysical contributions, the attention is focused on the brightness temperature increments relative to a reference, here set to the level obtained at $8 \mathrm{~m} \cdot \mathrm{s}^{-1}$. From 6 to $12 \mathrm{~m} \cdot \mathrm{s}^{-1}$, the brightness temperature increases by approximately $1.5 \mathrm{~K}$. At first order, it appears that the sensitivity to wind speed of the present empirical model is consistent with recent findings by [14] as shown in the dashed line. In a second step, the impact of variable SST and SSS distributions among the different datasets is evaluated using the dielectric model from KS77. Accounting for the slighly lower SST values in the 6 and 12 $\mathrm{m} \cdot \mathrm{s}^{-1}$ datasets - due to different geographical sampling during the data selection step, see Table I - it appears that the empirical one-parameter model is consistent with results in the literature on sensitivity to wind speed and 


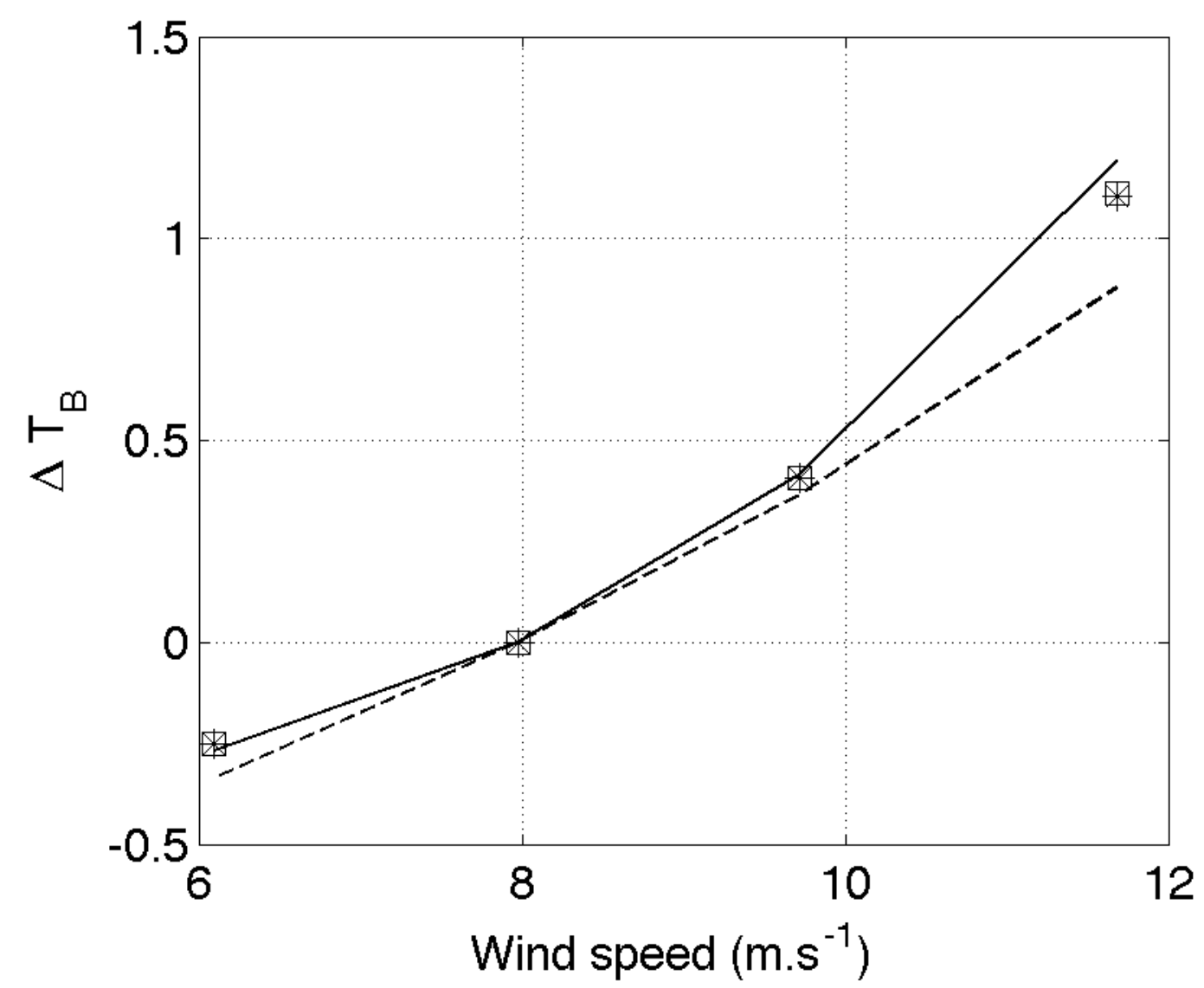

Fig. 4. Increment of brightness temperature with changing wind speed. Increments are provided relative to the value obtained with the $8 \mathrm{~m} \cdot \mathrm{s}^{-1}$ subset. Results are shown for $30^{\circ}$ incidence angle using the December 2011 dataset. Stars indicate the azimuthally-averaged value while squares correspond to the value from the simplified empirical model (see section III). The dashed line gives the roughness increment as predicted by [13] using the average wind speed from each subset. For the solid line, the prediction accounts for variations of surface dielectric conditions from one subset to the other; KS77's prediction and auxiliary SST and SSS information are used to estimate the impact of varying dielectric properties.

dielectric properties. The residual discrepancy is potentially due to imperfections in the empirical roughness model from [14]. The fitting procedure reproduces the geophysical variability well among the different datasets.

\section{B. Variability of the anomaly pattern}

1) Impact of geophysical conditions: The top row of Figure 5 shows the azimuthal anomaly pattern obtained for the $8 \mathrm{~m} \cdot \mathrm{s}^{-1}$ subset from the time periods of June 2011 (left) and December 2011 (right). The following rows show the departure of the $\{6,10,12\} \mathrm{m} \cdot \mathrm{s}^{-1}$ patterns from the $8 \mathrm{~m} \cdot \mathrm{s}^{-1}$ one used as reference.

In most cases, changing the geophysical conditions induces variations in the estimated pattern of amplitude smaller than $0.1 \mathrm{~K}$ with a root-mean-square $(\mathrm{rms})$ of $0.05 \mathrm{~K}$. The anomaly patterns are a factor of 10 larger than the difference patterns. For the June 2011 dataset, the differences between patterns essentially have a granular 


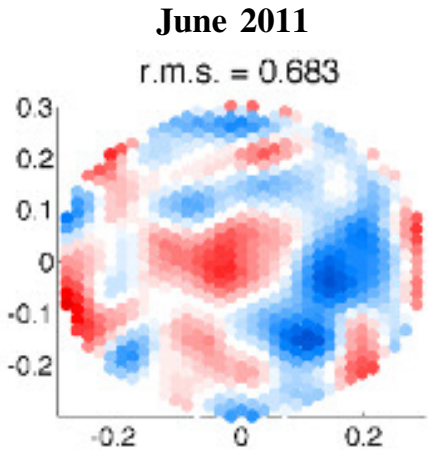

December 2011

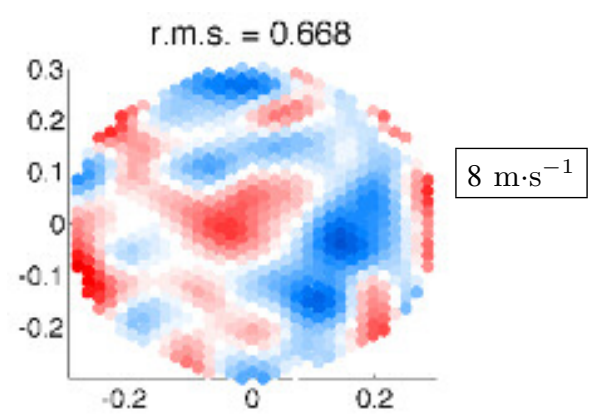

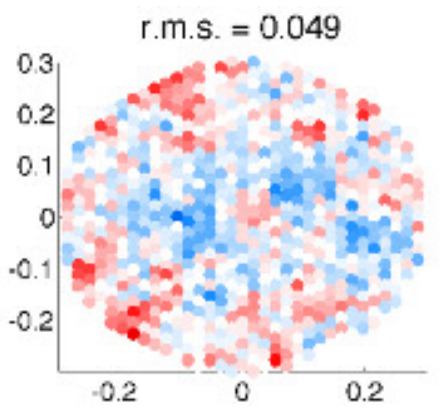
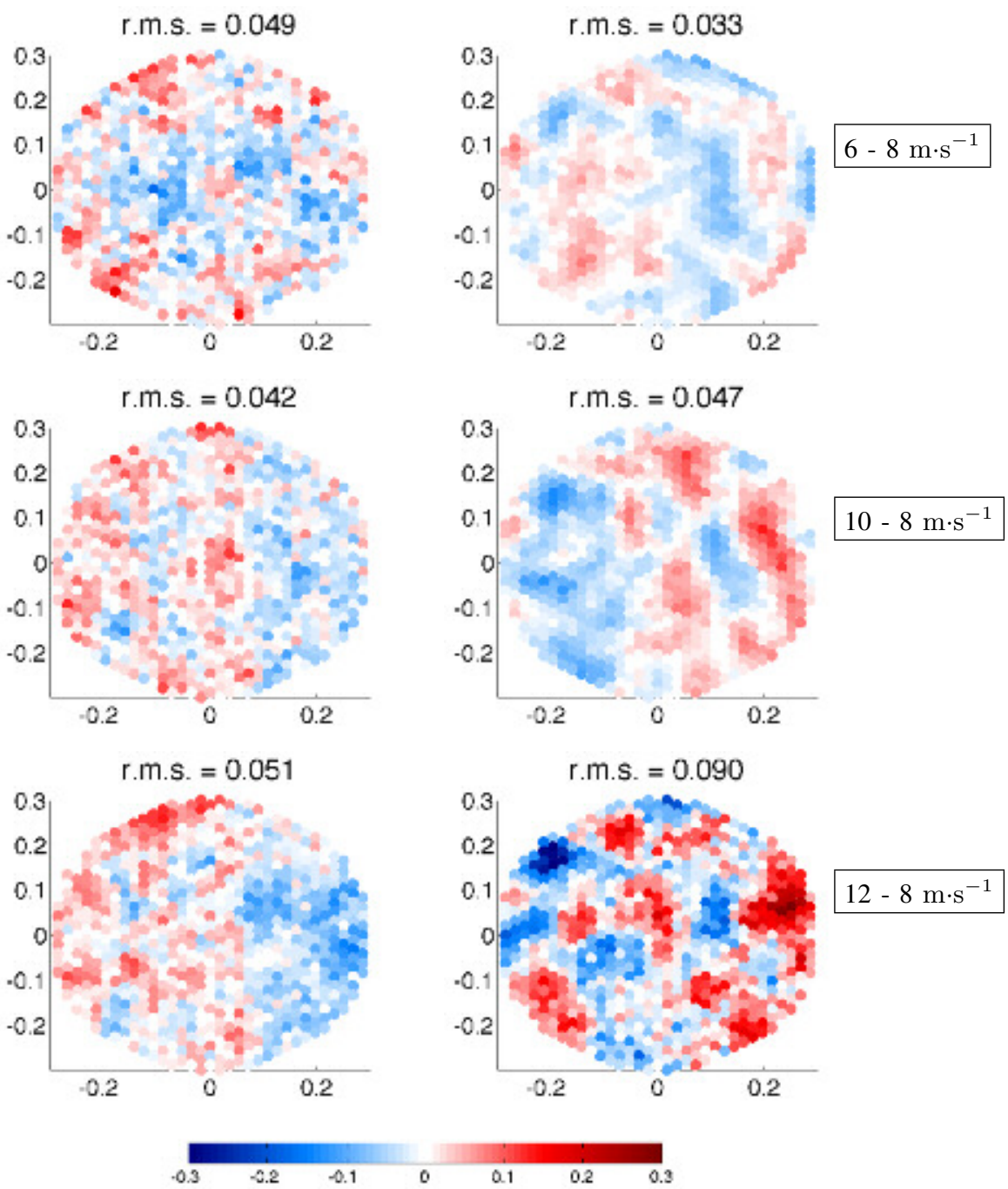

Fig. 5. Upper panels show the azimuthal anomaly pattern obtained for half of the first Stokes parameter and $8 \mathrm{~m} \cdot \mathrm{s}^{-1}$. Lower panels show the difference between the 6,10 and $12 \mathrm{~m} \cdot \mathrm{s}^{-1}$ patterns and the $8 \mathrm{~m} \cdot \mathrm{s}^{-1}$ one, and the rms difference calculated over the 0.3 circle. The left column refers to the June 2011 dataset, while the right column refers to the December 2011 dataset. 
structure, suggesting that they result from an incomplete reduction of noise present in the original data (due to a limited dataset). In a similar context, [12] performed a quantitative analysis of the residual noise level as a function of the number of samples used. The $0.05 \mathrm{~K} \mathrm{rms}$ value found here, jointly with the number of samples used (see Table I) is in very good agreement with their findings (see their Figure 2). In other cases (see right plots), the differences are rather structured, but are of a similarly small amplitude. Such cases probably provide evidence that azimuthally inhomogeneous variability is still present in the datasets, even after data selection, and provide a characteristic scale for the accuracy of the proposed methodology. For December 2011 and $12 \mathrm{~m} \cdot \mathrm{s}^{-1}$, the $\mathrm{rms}$ difference reaches $0.1 \mathrm{~K}$ for reasons that are not well understood. Using wind speed as a unique proxy for surface roughening is a reasonably robust assumption for most sea state conditions at any latitude. However, it is possible that, in cases of high wind speeds at low-to-middle latitudes, this assumption has a reduced validity if it does not account for sea state (and L-band effective roughness) variability under given wind speed conditions. If so, a refined data selection strategy could be investigated, but this is out of the scope of the present work. Here, it is simply used to help characterize the range of geophysical conditions under which the proposed methodology is applicable.

2) Temporal variability: The evaluation of the anomaly pattern temporal stability is now presented in a two-step approach. The attention is first focused on particular cases where residual geophysical variability is expected to be very similar so that it should cancel out in the relative analysis. Later, more general results are presented and discussed.

Comparison at one-year intervals: As both instrumental biases [11] and environmental contributions (such as celestial reflections, see Figure 1) are expected to be essentially functions of latitude and day of year, results from the same latitudinal band and with a one-year lag interval are first presented in Figure 6. Panels top-center and bottomleft correspond to the spatio-temporal windows proposed in section II II-A. All other panels correspond to spatiotemporal windows with higher expected celestial contaminations (see section II II-A). The top panels correspond to the latitude range of $\left[35^{\circ} \mathrm{S}, 0^{\circ} \mathrm{S}\right]$, while the bottom panels correspond to the latitude range of $\left[55^{\circ} \mathrm{S}, 35^{\circ} \mathrm{S}\right]$. The best consistency is observed between each top and corresponding bottom panel, and all patterns are clearly spatially structured. The difference patterns over a 1-year lag interval are essentially independent of latitude, suggesting that they are neither related to the instrumental biases evidenced in [11] nor related to residual celestial contaminations, both being highly latitudinally dependent. The difference patterns are likely a picture of differential residual errors from the visibility calibration - note that such differences never exceed $0.15 \mathrm{~K} \mathrm{rms}$ (see section I). Larger differences between left, center and right panels are consistent with the fact that differential residual calibration errors are expected to change from one time interval to the other (June, December, January). To summarize, the results presented in Figure 6 suggest that the methodology proposed in the present study permits estimation of a very stable azimuthal anomaly pattern when care is taken to avoid differential external source contaminations on average, and in the limit of unavoidable time-evolving visibility calibration errors (see section I).

Comparison at various time intervals: The anomaly patterns are now compared at various times of the year and in various latitudinal bands. Figure 7 shows pattern differences and rms values for the four dataset combinations. Clearly, when relaxing the time lag constraint, the patterns are less similar than when forcing 1-year time lag 


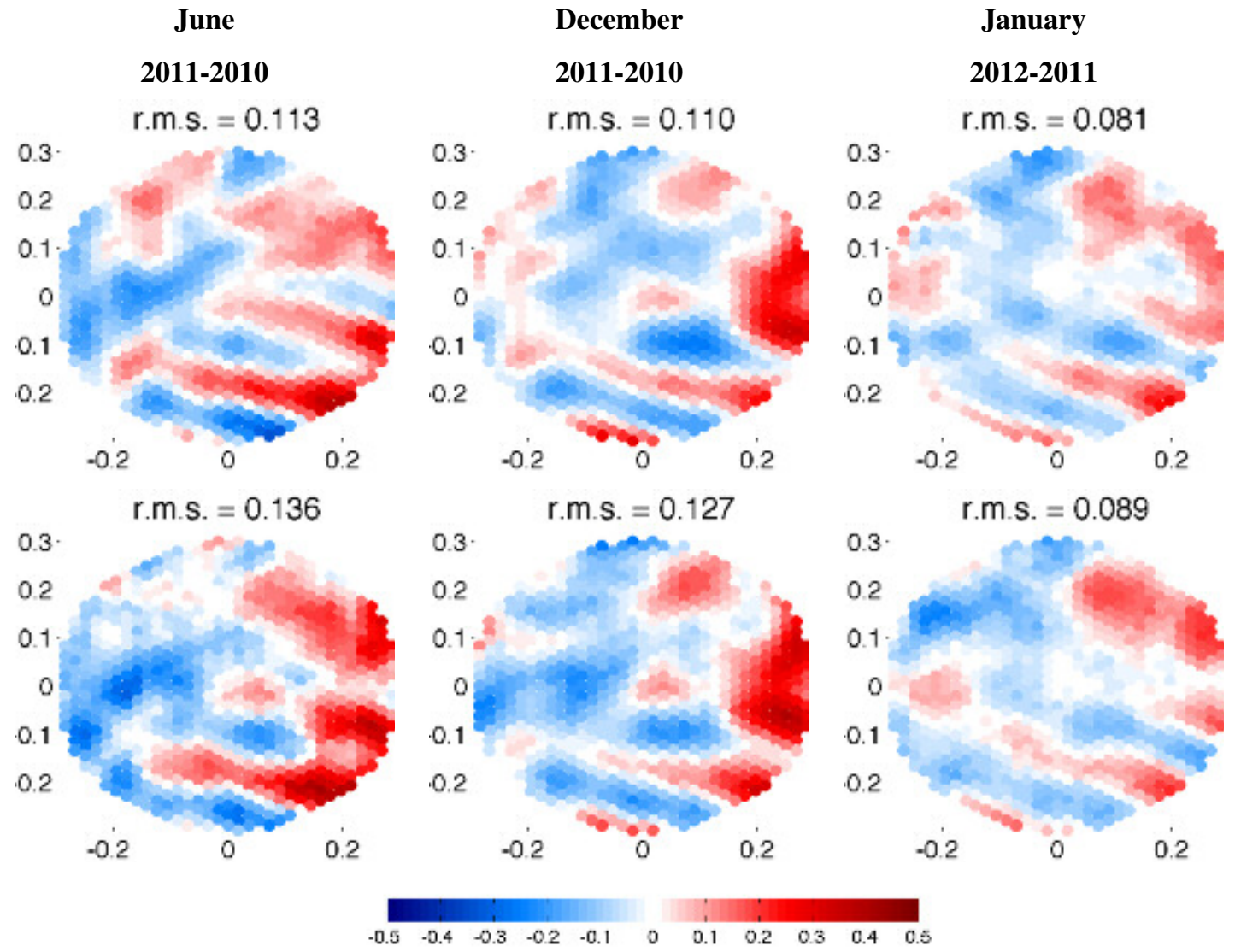

Fig. 6. Difference of azimuthal anomaly pattern at 1-year lag interval in the cos-dir antenna coordinate system for half of the first Stokes parameter. Upper and lower rows correspond respectively to the $\left[55^{\circ} \mathrm{S}-35^{\circ} \mathrm{S}\right]$ and $\left[35^{\circ} \mathrm{S}-0^{\circ} \mathrm{S}\right]$ latitude ranges. First column: June $10^{t h}-27^{t h}$; second: November $29^{t h}$ - December $16^{\text {th }}$; third: January $12^{\text {th }}-29^{t h}$.

and identical latitudinal range. The rms values are increased by 80 to $100 \%$. The comparison evidences that, in addition to the differential residual calibration errors, other factors contribute to the discrepancy between the estimated patterns. One of them is likely the spatio-temporal biases shown in [11] (see section I), which must contribute in a differential way. Indeed, such biases likely have a non-negligible component that vary with location in the antenna plane, which may contribute to the spatial patterns observed in Figure 7. Another factor may be the celestial residual contamination that, if not sufficiently reduced by the data selection criterion, may also appear here in a differential way. Reducing this second effect requires a refined data selection strategy based on a priori knowledge of the local celestial contamination in order to select observations individually rather than through a coarse geographical/temporal criterion. Considering the low threshold value that would need to be applied, and the impossibility of (presently) extending the time periods (because of spatio-temporal biases), such an approach would excessively reduce the number of observations available to estimate the patterns and, as a consequence, would lead to larger stability errors than those achieved in this study, i.e., $0.1 \mathrm{~K}$. 


\section{Dec'10 - June'10}

r.m.s. $=0.192$

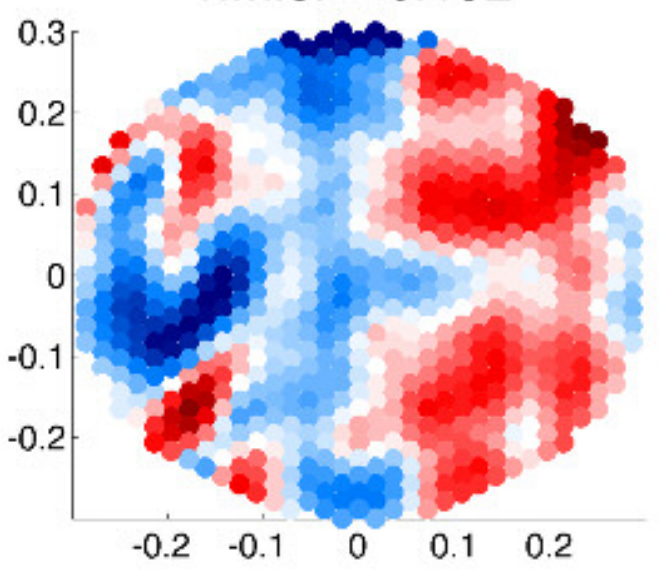

Dec'11 - June'11

r.m.s. $=0.195$

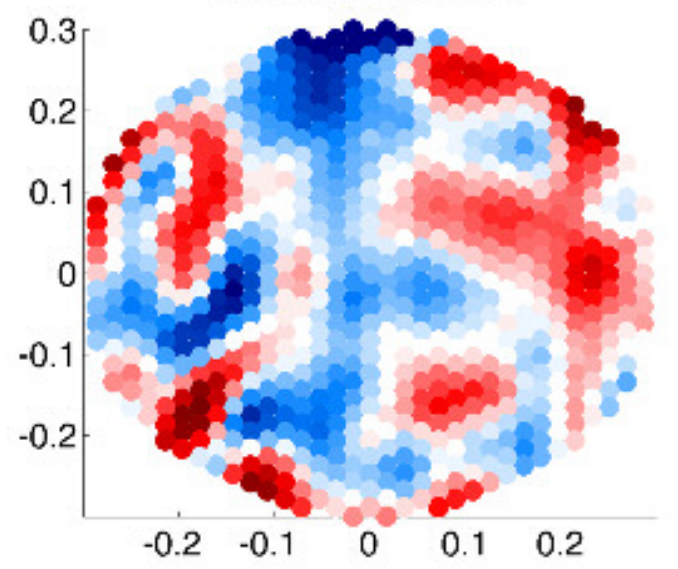

June'11 - Dec'10

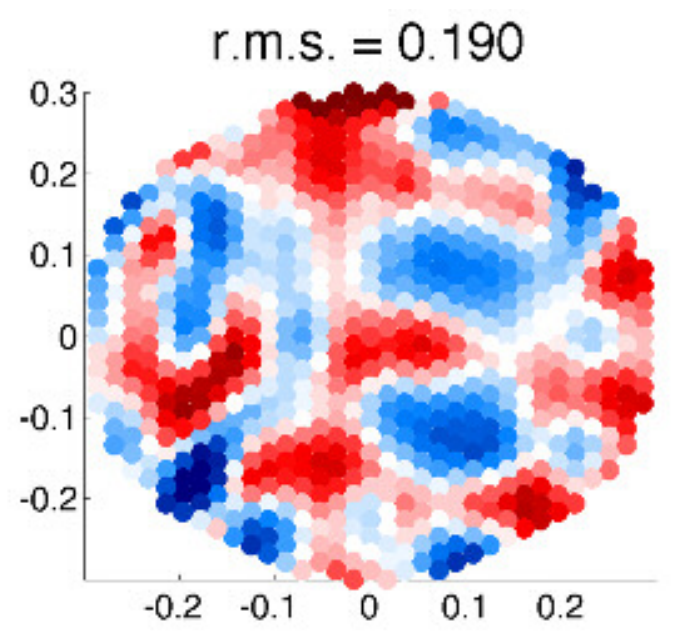

Dec'11 - June'10

r.m.s. $=0.235$

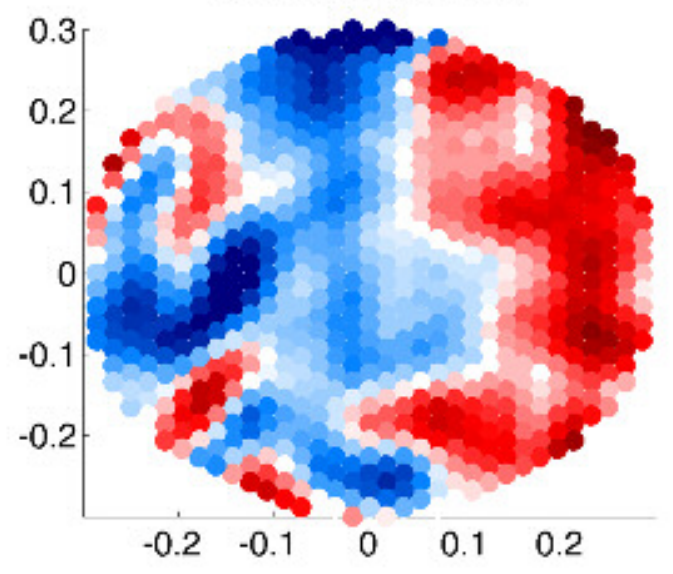

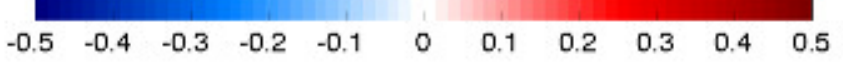

Fig. 7. Differences in azimuthal anomaly patterns between pairs of the four datasets corresponding to those defined in section II-A for 2010 and 2011. All results correspond to the half first Stokes parameter.

\section{Conclusion}

More than two and a half years after launch, ocean geophysical parameter retrieval from SMOS brightness temperature images has not yet achieved mission requirements. Strong spatio-temporal biases prevent consistent analysis of global datasets acquired at several week intervals. Characterization and mitigation of instrumental and model biases are of primary importance. The systematic error correction strategy implemented in the SMOS operational processing chain [9] accounts for the average discrepancy between $T_{B}$ measurements and modelled 
ones, thus mixing all kind of biases, such as those from the antenna patterns, antenna losses, foreign source corrections or the forward model used for geophysical parameter inversion. While practical for SSS retrieval for given versions of the calibration and forward model software, such a strategy may prevent consistent calibration error diagnostic or forward model improvement tasks. Furthermore, previous work [12] has shown that the corresponding correction pattern may vary by as much as $0.5 \mathrm{~K}$ when changing the dataset. An alternative strategy allowing separate characterization of all error sources and higher stability of the estimates is required.

The proposed approach permits diagnosis of the systematic (constant with time) $T_{B}$ errors in the antenna frame, with no impact of forward model errors. A specific data selection strategy and the use of an empirically fitted one-parameter (i.e. incidence angle dependent) $T_{B}$ model permits separation of the actual geophysical variability (caused by changing the selection criterion on dielectric and roughness conditions over the various datasets), which is essentially azimuth-independent, from the only-azimuth-dependent component of the instrumental errors. The latter is likely associated with the azimuthal $T_{B}$ anomalies induced by the antenna pattern errors through the reconstruction process. Furthermore, the robustness of the methodology is shown by the stability of the estimated anomaly patterns when computed from one dataset to another. The residual variability ranges from 0.03 to $0.14 \mathrm{~K}$ rms for all the comparisons, permitting sufficient control of the environmental conditions - essentially the celestial contamination.

Therefore, the stable patterns obtained in the present work may be applied to the SMOS $T_{B}(\mathrm{~S} 1)$ images to reduce the current azimuthal inconsistency between data obtained at different locations in the FOV, without modifying the data average absolute level and incidence angle dependence. It is a crucial capability both for future error diagnostic and forward model improvement tasks.

Future work should attempt to validate the estimated anomaly pattern in comparison with datasets acquired over non-oceanic targets, as antenna pattern errors should affect any image. As an example, emissivity from the Dome-C at Concordia station is known to be very stable over time, a characteristic which would greatly ease the necessary variability reduction. Further, as suggested by [13], the antenna pattern related $T_{B}$ errors may not be additive but rather multiplicative, i.e., they scale with the mean level of the images. This can actually be tested by applying the proposed methodology over ice, since ice emissivity is roughly twice as large as that of the ocean. The current methodology is fully compatible with a multiplicative formulation by further dividing the additive formulation of (4) by $T_{B}^{\text {model }}(\theta)$. Testing it over land targets is a possible but more complex task due to their high geophysical variability. Finally, results for only S1 have been presented in this work. Faraday rotation effects and imperfect knowledge of the total electron content (TEC) of the ionosphere introduce an additional source of error. On-going work deals with retrieving TEC from the MIRAS third Stokes parameter. Once the Faraday rotation is fully validated, the current methodology will be applied to the MIRAS X-pol and Y-pol data.

\section{ACKNOWLEDGEMENTS}

This work was supported by the Spanish National R+D Plan for the SMOS Barcelona Expert Center on Radiometric Calibration and Ocean Salinity activities, through project MIDAS-6 AYA2010-22062-C05 and previous 
grants.

\section{REFERENCES}

[1] J. Font, A. Camps, A. Borges, M. Martín-Neira, J. Boutin, N. Reul, Y. Kerr, A. Hahne, and S. Mecklenburg, "SMOS: The Challenging Sea Surface Salinity Measurement From Space," Proceedings of the IEEE, vol. 98, no. 5, pp. 649-665, May 2010.

[2] S. Mecklenburg, N. Wright, C. Bouzinac, and S. Delwart, "Getting down to business - SMOS operations and products," ESA Bulletin, vol. 137, p. 7, 2009.

[3] K. McMullan, M. Brown, M. Martín-Neira, W. Rits, S. Ekholm, J. Marti, and J. Lemanczyk, "SMOS: The payload," IEEE Trans. Geosci. Remote Sens., vol. 46, no. 3, pp. 594-605, 2008.

[4] M. Martín-Neira, M. Suess, J. Kainulainen, and F. Martin-Porqueras, "The flat target transformation," IEEE Trans. Geosci. Remote Sens., vol. 46, no. 3, pp. 613-620, 2008.

[5] E. Anterrieu and A. Khazaal, "Brightness temperature map reconstruction from dual-polarimetric visibilities in synthetic aperture imaging radiometry," IEEE Trans. Geosci. Remote Sens., vol. 46, no. 3, pp. 606-612, march 2008.

[6] J. Tenerelli, N. Reul, and B. Chapron, "Analysis of SMOS brightness temperatures obtained from March through May 2010," in Proc. ESA Living Planet Symp., Bergen, Norway, Jun. 28Jul. 2, ESA Special Publication SP-686, 2010.

[7] A. Camps, M. Vall-llossera, L. Batres, F. Torres, N. Duffo, and I. Corbella, "Retrieving sea surface salinity with multiangular L-band brightness temperatures: Improvement by spatiotemporal averaging," Radio Sci., vol. 40, no. 2, pp. 292-295, Mar. 2005. [Online]. Available: http://dx.doi.org/10.1029/2004RS003040

[8] E. Anterrieu, "On the reduction of the reconstruction bias in synthetic aperture imaging radiometry," IEEE Trans. Geosci. Remote Sens., vol. 45, no. 3, pp. 592 -601, Mar 2007.

[9] J. Font, J. Boutin, N. Reul, P. Spurgeon, J. Ballabrera-Poy, A. Chuprin, C. Gabarro, J. Gourrion, C. Henocq, S. Lavender, N. Martin, J. Martinez, M. McCulloch, I. Meirold-Mautner, C. Mugerin, F. Petitcolin, M. Portabella, R. Sabia, M. Talone, T. J., A. Turiel, J. Vergely, P. Waldteufel, X. Yin, S. Zine, and S. Delwart, "SMOS first data analysis for sea surface salinity determination," Int. J. Remote Sens., 2012, accepted for publication.

[10] N. Reul, J. Tenerelli, J. Boutin, B. Chapron, F. Paul, E. Brion, F. Gaillard, and O. Archer, "Overview of the first SMOS Sea Surface Salinity products. part i: quality assessment for the second half of 2010," IEEE Trans. Geosci. Remote Sens., vol. 50, no. 5, pp. 1636-1647, May 2012.

[11] R. Oliva, M. Martín-Neira, I. Corbella, F. Torres, J. Kainulainen, J. Tenerelli, F. Cabot, and F. Martin-Porqueras, "SMOS calibration and instrument performance after one year in orbit," IEEE Trans. Geosci. Remote Sens., vol. 51, no. 1, pp. 654-670, 2012.

[12] J. Gourrion, R. Sabia, M. Portabella, J. Tenerelli, S. Guimbard, and A. Camps, "Characterization of the SMOS instrumental error pattern correction over the ocean," IEEE Geosci. Remote Sens. Lett., vol. 9, no. 4, pp. 793-797, July 2012.

[13] F. Torres, I. Corbella, L. Wu, N. Duffo, J. Gourrion, J. Font, and M. Martín-Neira, "Minimization of image distortion in SMOS brightness temperature maps over the ocean," IEEE Geosci. Remote Sens. Lett., vol. 9, no. 1, pp. 18-22, Jan 2012. [Online]. Available: http://ieeexplore.ieee.org/lpdocs/epic03/wrapper.htm?arnumber=5940991

[14] S. Guimbard, J. Gourrion, M. Portabella, A. Turiel, C. Gabarró, and J. Font, "SMOS semi-empirical ocean forward model adjustment," IEEE Trans. Geosci. Remote Sens., 2012.

[15] L. A. Klein and C. T. Swift, "An improved model for the dielectric constant of sea water at microwave frequencies," IEEE Trans. Antennas Propag., vol. 25, no. 1, pp. 104-111, Jan 1977. 